\title{
Synchronization of Chaotic Systems Using Time-Delayed Fuzzy State-Feedback Controller
}

\author{
H. K. Lam, Member, IEEE, Wing-Kuen Ling, Herbert Ho-Ching Iu, Senior Member, IEEE, and \\ Steve S. H. Ling, Member, IEEE
}

\begin{abstract}
This paper presents the fuzzy-model-based control approach to synchronize two chaotic systems subject to parameter uncertainties. A fuzzy state-feedback controller using the system state of response chaotic system and the time-delayed system state of drive chaotic system is employed to realize the synchronization. The time delay which complicates the system dynamics makes the analysis difficult. To investigate the system stability and facilitate the design of fuzzy controller, Takagi-Sugeno (T-S) fuzzy models are employed to represent the system dynamics of the chaotic systems. Furthermore, the membership grades of the T-S fuzzy models become uncertain due to the existence of parameter uncertainties which further complicates the system analysis. To ease the stability analysis and produce less conservative analysis result, the membership functions of both T-S fuzzy models and fuzzy controller are considered. Stability conditions are derived using Lyapunov-based approach to aid the design of fuzzy state-feedback controller to synchronize the chaotic systems. Simulation examples are presented to illustrate the merits of the proposed approach.
\end{abstract}

Index Terms-Chaotic synchronization, fuzzy control, stability, Takagi-Sugeno (T-S) fuzzy model.

\section{INTRODUCTION}

$\mathbf{F}$ UZZY-MODEL-based control approach is a promising approach to deal with complex nonlinear systems. It has been successfully applied in various applications. Recently, fuzzymodel-based control approach has been employed to synchronize chaotic systems, which is a useful application in communication system to ensure a secure communication.

In fuzzy-model-based control approach, generally, TakagiSugeno (T-S) fuzzy model [1], [2] is employed to describe the dynamical behaviors of the response and drive chaotic systems. It was shown in [3]-[5] that most common chaotic systems can be represented by T-S fuzzy models with simple rules. Based on the T-S fuzzy model, a fuzzy state-feedback controller [3]-[6] is then designed to realize the synchronization. Under a design criterion that the grades of membership of both response and drive chaotic system are known, linear-matrix-inequality (LMI)-based exact linearization conditions [3], [6] were given to design a fuzzy state-feedback controller to synchronize two

Manuscript received February 25, 2007; revised June 25, 2007. This work was supported by the Division of Engineering, King's College London, London, U.K. This paper was recommended by Associate Editor C.-W. Wu.

H. K. Lam and W.-K. Ling are with the Division of Engineering, King's College London, London WC2R 2LS, U. K. (e-mail: hak-keung.lam@kcl.ac.uk).

H. H.-C. Iu and S. S. H. Ling are with the School of Electrical, Electronic and Computer Engineering, The University of Western Australia, Perth, WA 6009, Australia.

Digital Object Identifier 10.1109/TCSI.2008.916430 identical chaotic systems. In [4], [5], this design criterion was alleviated by using the $H_{\infty}$ tracking control approach. Under the approach in [4], [5], the grades of membership of the drive chaotic system are not necessarily known and the tracking performance is guaranteed by an $H_{\infty}$ tracking performance index. The fuzzy-model-based control approach has combined with adaptive ability [6]-[11] to deal with chaotic systems subject to parameter uncertainties. With the outstanding approximation ability of the fuzzy system, the uncertain parameter values of the chaotic systems can be estimated in an online manner according to some update rules. A fuzzy controller can generate an appropriate control action based on the estimated parameters. The adaptive fuzzy approach offers a superior robustness property, however, computational demand and structural complexity of the controller are increased. In some operating environment, the system state information of the drive chaotic system reaches the responses system with time delay owing to the long-distance transmission. Under such a situation, the current state information of the drive chaotic system cannot be obtained to realize the synchronization. In [12]-[14], synchronization using time-delayed feedback control was investigated. Linear controller using constant time-delayed system state information of both drive and response chaotic system, and the current system state information of response chaotic system was proposed to realize the synchronization. Both time-delay independent and dependent stability conditions were derived [12]-[14] using the Lyapunov-Krasovksii function. This delayed-feedback control approach was extended to adaptive fuzzy framework [15]. In [17]-[19], the synchronization of neural networks subject to time delay was considered.

In this paper, a fuzzy controller is proposed to synchronize two chaotic systems. The fuzzy controller makes use of current system state information of the response chaotic system and the time-delayed system state information of the drive chaotic system to realize the synchronization. The time delay to be considered is time varying and uncertain in value. It is due to this reason, the proposed fuzzy state-feedback controller cannot use the time-delayed system state information of the response chaotic system compared to the linear control [12]-[14] and the adaptive fuzzy control [15] approaches of which constant time delay was considered. To cope with the time-varying delay, the boundedness property of the system states of the drive chaotic system is taken advantage to investigate system stability. Furthermore, the parameter uncertainties of the chaotic systems eliminate the favourable properties of the fuzzy-model-based control approach to facilitate the stability analysis and produce relaxed stability conditions [3]-[6], [12]-[15]. To alleviate the 


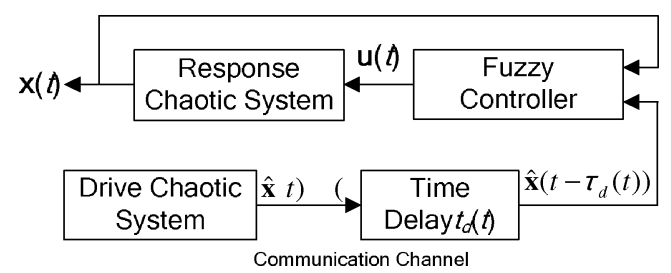

Fig. 1. Block diagram of the chaotic synchronization system.

difficulties introduced by parameter uncertainties, membership functions of both fuzzy model and fuzzy controller are considered. Consequently, some free matrices are allowed to be introduced to the stability conditions to ease the stability analysis and produce less conservative stability conditions. LMI-based stability conditions are derived to aid the design of a fuzzy controller to realize the synchronization.

This paper is organized as follows. In Section II, the fuzzy model and the time-delayed fuzzy state-feedback controller is presented. In Section III, system stability is investigated. LMIbased stability conditions are derived to guarantee the system stability. In Section IV, a simulation example is given to illustrate the effectiveness of the proposed approach. A conclusion is drawn in Section V.

\section{FuzZy Model AND Time-Delayed FuZZy Controller}

In this paper, we consider the scenario depicted in the block diagram in Fig. 1, e.g., a communication channel. Referring to this block diagram, in the remote side, we have a drive chaotic system subject to parameter uncertainties. The system state $\hat{\mathbf{x}}(t)$ of the drive chaotic system is transmitted over a channel to the other end. The long distance of the communication channel will introduces a time-varying delay of $\tau_{d}(t) \geq 0$ to $\hat{\mathbf{x}}(t)$. As a result, $\hat{\mathbf{x}}\left(t-\tau_{d}(t)\right)$ is available for synchronization. As the fuzzy controller is assumed to be very close to the response system subject to parameter uncertainties, hence, the time delay for the response chaotic system with the system state of $\mathbf{x}(t)$ is insignificant. Consequently, the fuzzy controller realizes the synchronization based on the timed-delayed system state of $\hat{\mathbf{x}}\left(t-\tau_{d}(t)\right)$ and current system state of $\mathbf{x}(t)$.

To facilitate the system analysis and controller synthesis, fuzzy models are employed to represent the dynamical behavior of the response and drive chaotic systems subject to parameter uncertainties. A time-delayed fuzzy state-feedback controller is designed accordingly to drive the system state of the response chaotic system to follow those of the drive chaotic system. The details of the fuzzy model and fuzzy controller are presented in the following subsections.

\section{A. Fuzzy Model}

Let $p$ be the number of fuzzy rules describing the chaotic system subject to parameter uncertainties with control input term. The $i$ th rule is of the following format:

Rule $i$ :IF $f_{1}(\mathbf{x}(t))$ is $\mathrm{M}_{1}^{i}$ AND... AND $f_{\Psi}(\mathbf{x}(t))$ is $\mathrm{M}_{\Psi}^{i}$ THEN $\dot{\mathbf{x}}(t)=\mathbf{A}_{i} \mathbf{x}(t)+\mathbf{B u}(t), \quad i=1,2, \ldots, p$ where $\mathrm{M}_{\alpha}^{i}$ is a fuzzy term of rule $i$ corresponding to the function $f_{\alpha}(\mathbf{x}(t))$ with known form, $\alpha=1,2, \ldots, \Psi, i=1,2, \ldots, p$, $\Psi$ is a positive integer; $\mathbf{x}(t) \in \Re^{n \times 1}$ is the system state vector; $\mathbf{A}_{i} \in \Re^{n \times n}$ and $\mathbf{B} \in \Re^{n \times m}$ are the known constant system and input matrices, respectively; $\mathbf{u}(t) \in \Re^{m \times 1}$ is the input vector. The system dynamics are described by

$$
\dot{\mathbf{x}}(t)=\sum_{i=1}^{p} w_{i}(\mathbf{x}(t))\left(\mathbf{A}_{i} \mathbf{x}(t)+\mathbf{B u}(t)\right)
$$

where

$$
\begin{aligned}
& \sum_{i=1}^{p} w_{i}(\mathbf{x}(t))=1, w_{i}(\mathbf{x}(t)) \in\left[\begin{array}{ll}
0 & 1
\end{array}\right], \quad \text { for all } i \\
& w_{i}(\mathbf{x}(t)) \\
& =\frac{\mu_{\mathrm{M}_{1}^{i}}\left(f_{1}(\mathbf{x}(t))\right) \times \mu_{\mathrm{M}_{2}^{i}}\left(f_{2}(\mathbf{x}(t))\right) \times \cdots \times \mu_{\mathrm{M}_{\Psi}^{i}}\left(f_{\Psi}(\mathbf{x}(t))\right)}{\sum_{k=1}^{p}\left(\mu_{\mathrm{M}_{1}^{k}}\left(f_{1}(\mathbf{x}(t))\right) \times \mu_{\mathrm{M}_{2}^{k}}\left(f_{2}(\mathbf{x}(t))\right) \times \cdots \times \mu_{\mathrm{M}_{\Psi}^{k}}\left(f_{\Psi}(\mathbf{x}(t))\right)\right)}
\end{aligned}
$$

is a nonlinear function of $\mathbf{x}(t)$ and $\mu_{\mathrm{M}_{\alpha}^{i}}\left(f_{\alpha}(\mathbf{x}(t))\right)$ is the grade of membership corresponding to the fuzzy terms $\mathrm{M}_{\alpha}^{i}$. Let the chaotic system of (2) be the response system. Similarly, the dynamics of the drive chaotic system subject to parameter uncertainties can be represented by a fuzzy model with $q$ fuzzy rules in the form of (1). Consequently, the dynamics of the drive chaotic system can be represented as

$$
\dot{\hat{\mathbf{x}}}(t)=\sum_{j=1}^{q} \hat{w}_{j}(\hat{\mathbf{x}}(t)) \hat{\mathbf{A}}_{j} \hat{\mathbf{x}}(t)
$$

where $\hat{\mathbf{x}}(t) \in \Re^{n \times 1}$ is the system state vector. $\hat{\mathbf{A}}_{j} \in \Re^{n \times n}$ is the known constant system matrix; $\hat{w}_{j}(\hat{\mathbf{x}}(t)) \in\left[\begin{array}{ll}0 & 1\end{array}\right]$ is the grade of the membership and $\sum_{j=1}^{q} \hat{w}_{j}(\hat{\mathbf{x}}(t))=1$. It should be noted that there is no control input term for the drive chaotic system. The grades of membership are uncertain in value due to the existence of the parameter uncertainties.

\section{B. Time-Delayed Fuzzy State-Feedback Controller}

A time-delayed fuzzy controller with $p$ fuzzy rules is employed to realize the synchronization. The $j$ th rule of the fuzzy controller is of the following format:

$$
\begin{array}{r}
\text { Rule } j: \operatorname{IF} g_{1}(\mathbf{x}(t)) \text { is } \mathrm{N}_{1}^{j} \text { AND } \ldots \text { AND } g_{\Omega}(\mathbf{x}(t)) \text { is } \mathrm{N}_{\Omega}^{j} \\
\text { THEN } \mathbf{u}(t)=\mathbf{G}_{j}\left(\mathbf{x}(t)-\hat{\mathbf{x}}\left(t-\tau_{d}(t)\right), \quad j=1,2, \ldots, p\right.
\end{array}
$$

where $\mathrm{N}_{\beta}^{j}$ is a fuzzy term of rule $j$ corresponding to the function $g_{\beta}(\mathbf{x}(t)), \beta=1,2, \ldots, \Omega ; j=1,2, \ldots, p ; \Omega$ is a positive integer; $\mathbf{G}_{j} \in \Re^{m \times n}$ is the feedback gain of rule $j$ to be designed; $\tau_{d}(t) \geq 0$ denotes the uncertain time-varying time delay. The inferred time-delayed fuzzy controller is defined as,

$$
\mathbf{u}(t)=\sum_{j=1}^{p} m_{j}(\mathbf{x}(t)) \mathbf{G}_{j}\left(\mathbf{x}(t)-\hat{\mathbf{x}}\left(t-\tau_{d}(t)\right)\right)
$$


where

$$
\begin{aligned}
& \sum_{j=1}^{p} m_{j}(\mathbf{x}(t))=1, m_{j}(\mathbf{x}(t)) \in\left[\begin{array}{ll}
0 & 1
\end{array}\right], \text { for all } j \\
& m_{j}(\mathbf{x}(t)) \\
& =\frac{\mu_{\mathrm{N}_{1}^{j}}\left(g_{1}(\mathbf{x}(t))\right) \times \mu_{\mathrm{N}_{2}^{j}}\left(g_{2}(\mathbf{x}(t))\right) \times \cdots \times \mu_{\mathrm{N}_{\Omega}^{j}}\left(g_{\Omega}(\mathbf{x}(t))\right)}{\sum_{k=1}^{p}\left(\mu_{\mathrm{N}_{1}^{k}}\left(g_{1}(\mathbf{x}(t))\right) \times \mu_{\mathrm{N}_{2}^{k}}\left(g_{2}(\mathbf{x}(t))\right) \times \cdots \times \mu_{\mathrm{N}_{\Omega}^{k}}\left(g_{\Omega}(\mathbf{x}(t))\right)\right)}
\end{aligned}
$$

is a nonlinear function of $\mathbf{x}(t)$ and $\mu_{\mathrm{N}_{\beta}^{j}}\left(g_{\beta}(\mathbf{x}(t))\right)$ is the grade of membership corresponding to the fuzzy term $\mathrm{N}_{\beta}^{j}$. Referring to the proposed fuzzy controller of (7), it is assumed that the system state $\mathbf{x}(t)$ of the response chaotic system in the local side can be accessed without time delay. However, due to the long transmission line, the system state $\hat{\mathbf{x}}\left(t-\tau_{d}(t)\right)$ of the drive chaotic system in the remote side is subject to an uncertain timevarying delay $\tau_{d}(t)$.

\section{Stability ANALYSIS}

The objective of synchronization is to drive the system states of the response chaotic system of (2) to follow those of the drive chaotic system of (5) using the time-delayed fuzzy controller of (7). To proceed to the system stability analysis, from (2), (5) and (7), the error system is defined as follows:

$$
\begin{aligned}
\dot{\mathbf{e}}(t)= & \dot{\mathbf{x}}(t)-\dot{\hat{\mathbf{x}}}(t) \\
= & \sum_{i=1}^{p} w_{i}(\mathbf{x}(t))\left(\mathbf{A}_{i} \mathbf{x}(t)+\mathbf{B u}(t)\right)-\sum_{j=1}^{q} \hat{w}_{j}(\hat{\mathbf{x}}(t)) \hat{\mathbf{A}}_{j} \hat{\mathbf{x}}(t) \\
= & \sum_{i=1}^{p} w_{i}(\mathbf{x}(t)) \mathbf{A}_{i} \mathbf{e}(t)+\sum_{i=1}^{p} m_{i}(\mathbf{x}(t)) \mathbf{B} \mathbf{G}_{i} \mathbf{e}(t) \\
& +\sum_{i=1}^{p} w_{i}(\mathbf{x}(t)) \mathbf{A}_{i} \hat{\mathbf{x}}(t) \\
& +\sum_{i=1}^{p} m_{i}(\mathbf{x}(t)) \mathbf{B G}_{i}\left(\hat{\mathbf{x}}(t)-\hat{\mathbf{x}}\left(t-\tau_{d}(t)\right)\right) \\
& -\sum_{j=1}^{q} \hat{w}_{j}(\hat{\mathbf{x}}(t)) \hat{\mathbf{A}}_{j} \hat{\mathbf{x}}(t) \\
= & \sum_{i=1}^{p} \sum_{j=1}^{p} w_{i}(\mathbf{x}(t)) m_{j}(\mathbf{x}(t))\left(\mathbf{A}_{i}+\mathbf{B G}_{j}\right) \mathbf{e}(t)+\mathbf{m}_{e}(t)
\end{aligned}
$$

where

$$
\begin{aligned}
\mathbf{m}_{e}(t)= & \sum_{i=1}^{p} w_{i}(\mathbf{x}(t)) \mathbf{A}_{i} \hat{\mathbf{x}}(t) \\
& +\sum_{i=1}^{p} m_{i}(\mathbf{x}(t)) \mathbf{B} \mathbf{G}_{i}\left(\hat{\mathbf{x}}(t)-\hat{\mathbf{x}}\left(t-\tau_{d}(t)\right)\right) \\
& -\sum_{j=1}^{q} \hat{w}_{j}(\hat{\mathbf{x}}(t)) \hat{\mathbf{A}}_{j} \hat{\mathbf{x}}(t)
\end{aligned}
$$

It should be noted that $\mathbf{m}_{e}(t)$ is bounded due to $\hat{\mathbf{x}}(t), w_{i}(\mathbf{x}(t))$ and $\hat{w}_{j}(\hat{\mathbf{x}}(t))$ are bounded. In the following analysis, $w_{i}(\mathbf{x}(t))$, $m_{j}(\mathbf{x}(t))$ and $\hat{w}_{j}(\hat{\mathbf{x}}(t))$ are denoted as $w_{i}, m_{j}$ and $\hat{w}_{j}$ for simplicity. Furthermore, the property of the membership functions that $\sum_{i=1}^{p} w_{i}=\sum_{j=1}^{p} m_{j}=\sum_{i=1}^{p} \sum_{j=1}^{p} w_{i} m_{j}=1$ is applied in the following analysis. The error system of (10) is represented as the following form to facilitate the stability analysis:

$$
\begin{aligned}
\dot{\mathbf{e}}(t) & =\mathbf{z}(t) \\
\mathbf{z}(t) & =\sum_{i=1}^{p} w_{i} \mathbf{A}_{i} \mathbf{e}(t)+\mathbf{y}(t)+\mathbf{m}_{e}(t) \\
\mathbf{y}(t) & =\sum_{i=1}^{p} m_{i} \mathbf{B G}_{i} \mathbf{e}(t) .
\end{aligned}
$$

From (12) and (13), the following property, which is applied during the stability analysis, can be obtained:

$$
\sum_{i=1}^{p} \sum_{j=1}^{p} w_{i} m_{j}\left[\begin{array}{cccc}
\mathbf{0} & \mathbf{0} & \mathbf{0} & \mathbf{0} \\
\mathbf{0} & \mathbf{0} & \mathbf{0} & \mathbf{0} \\
\mathbf{B} \mathbf{G}_{j} & \mathbf{0} & -\mathbf{I} & \mathbf{0} \\
\mathbf{A}_{i} & \mathbf{I} & \mathbf{I} & -\mathbf{I}
\end{array}\right]\left[\begin{array}{c}
\mathbf{e}(t) \\
\mathbf{m}_{e}(t) \\
\mathbf{y}(t) \\
\mathbf{z}(t)
\end{array}\right]=\left[\begin{array}{l}
\mathbf{0} \\
\mathbf{0} \\
\mathbf{0} \\
\mathbf{0}
\end{array}\right]
$$

To investigate the stability of (10), the following Lyapunov function candidate is employed.

$$
V(t)=\mathbf{e}(t)^{\mathrm{T}} \mathbf{P}_{1} \mathbf{e}(t)
$$

where $\mathbf{P}_{1}=\mathbf{P}_{1}^{\mathrm{T}} \in \Re^{n \times n}>0$. From (11) and (15), we have

$$
\begin{aligned}
\dot{V}(t)= & \mathbf{e}(t)^{\mathrm{T}} \mathbf{P}_{1} \dot{\mathbf{e}}(t)+\dot{\mathbf{e}}(t)^{\mathrm{T}} \mathbf{P}_{1} \mathbf{e}(t) \\
= & \mathbf{e}(t)^{\mathrm{T}} \mathbf{P}_{1} \mathbf{z}(t)+\mathbf{z}(t)^{\mathrm{T}} \mathbf{P}_{1} \mathbf{e}(t) \\
= & {\left[\begin{array}{c}
\mathbf{e}(t) \\
\mathbf{m}_{e}(t) \\
\mathbf{y}(t) \\
\mathbf{z}(t)
\end{array}\right]^{\mathrm{T}}\left(\mathbf{P}^{\mathrm{T}}\left[\begin{array}{llll}
\mathbf{0} & \mathbf{0} & \mathbf{0} & \mathbf{I} \\
\mathbf{0} & \mathbf{0} & \mathbf{0} & \mathbf{0} \\
\mathbf{0} & \mathbf{0} & \mathbf{0} & \mathbf{0} \\
\mathbf{0} & \mathbf{0} & \mathbf{0} & \mathbf{0}
\end{array}\right]\right.} \\
& \left.+\left[\begin{array}{llll}
\mathbf{0} & \mathbf{0} & \mathbf{0} & \mathbf{I} \\
\mathbf{0} & \mathbf{0} & \mathbf{0} & \mathbf{0} \\
\mathbf{0} & \mathbf{0} & \mathbf{0} & \mathbf{0} \\
\mathbf{0} & \mathbf{0} & \mathbf{0} & \mathbf{0}
\end{array}\right]^{\mathrm{T}} \mathbf{P}\right)\left[\begin{array}{c}
\mathbf{e}(t) \\
\mathbf{m}_{e}(t) \\
\mathbf{y}(t) \\
\mathbf{z}(t)
\end{array}\right]
\end{aligned}
$$

where

$$
\mathbf{P}=\left[\begin{array}{cccc}
\mathbf{P}_{1} & \mathbf{0} & \mathbf{0} & \mathbf{0} \\
\mathbf{P}_{2} & \mathbf{P}_{3} & \mathbf{0} & \mathbf{0} \\
\mathbf{P}_{4} & \mathbf{P}_{5} & \mathbf{P}_{6} & \mathbf{0} \\
\mathbf{P}_{7} & \mathbf{P}_{8} & \mathbf{P}_{9} & \mathbf{P}_{10}
\end{array}\right]
$$

and $\hat{\mathbf{P}}_{k} \in \Re^{n \times n}, k=1,2, \ldots, 10$. From (14) and (16), we have (17), shown at the bottom of the next page, where $\mathbf{U}=\mathbf{U}^{\mathrm{T}}=$ $\sum_{i=1}^{p} w_{i} \mathbf{U}_{i} \in \Re^{n \times n}, \mathbf{U}_{i}=\mathbf{U}_{i}^{\mathrm{T}} \in \Re^{n \times n}>0, i=1,2, \ldots, p$ and $\sigma$ is a nonzero positive scalar. Let

$$
\begin{aligned}
\mathbf{X} & =\left[\begin{array}{cccc}
\mathbf{X}_{1} & \mathbf{0} & \mathbf{0} & \mathbf{0} \\
\mathbf{X}_{1} & \mathbf{X}_{1} & \mathbf{0} & \mathbf{0} \\
\mathbf{X}_{2} & \mathbf{X}_{3} & \mathbf{X}_{4} & \mathbf{0} \\
\mathbf{X}_{5} & \mathbf{X}_{6} & \mathbf{X}_{7} & \mathbf{X}_{8}
\end{array}\right] \\
& =\mathbf{P}^{-1} \\
\mathbf{X}_{1} & =\mathbf{X}_{1}^{\mathrm{T}}=\mathbf{P}_{1}^{-1} \in \Re^{n \times n} \\
\mathbf{X}_{k} & =\sum_{i=1}^{p} w_{i} \mathbf{X}_{k i} \in \Re^{n \times n}
\end{aligned}
$$


and $\mathbf{X}_{k i} \in \Re^{n \times n}, i=1,2, \ldots, p ; k=2,3, \ldots, 8$,

$$
\begin{aligned}
\mathbf{v}(t) & =\mathbf{X}^{-1}\left[\begin{array}{c}
\mathbf{e}(t) \\
\mathbf{m}_{e}(t) \\
\mathbf{y}(t) \\
\mathbf{z}(t)
\end{array}\right] \\
\mathbf{G}_{i} & =\mathbf{N}_{i} \mathbf{X}_{1}^{-1}
\end{aligned}
$$

and $\hat{\mathbf{N}}_{i} \in \Re^{m \times n}, i=1,2, \ldots, p$. From (17), we have (18), shown at the bottom of the page, where the third equation at the bottom of the page holds. The symbol "*" denotes the transposed element at the corresponding position. It can be seen from (18) that the system is stable if $\mathbf{V}_{i j}<0$ for all $i$ and $j$. However, it produces a very conservative stability analysis result. In order to alleviate the conservativeness, the membership functions of both fuzzy model and fuzzy controller are designed such that

$$
\begin{aligned}
& \dot{V}(t)=\left[\begin{array}{c}
\mathbf{e}(t) \\
\mathbf{m}_{e}(t) \\
\mathbf{y}(t) \\
\mathbf{z}(t)
\end{array}\right]^{\mathrm{T}}\left(\mathbf{P}^{\mathrm{T}}\left[\begin{array}{llll}
\mathbf{0} & \mathbf{0} & \mathbf{0} & \mathbf{I} \\
\mathbf{0} & \mathbf{0} & \mathbf{0} & \mathbf{0} \\
\mathbf{0} & \mathbf{0} & \mathbf{0} & \mathbf{0} \\
\mathbf{0} & \mathbf{0} & \mathbf{0} & \mathbf{0}
\end{array}\right]+\left[\begin{array}{llll}
\mathbf{0} & \mathbf{0} & \mathbf{0} & \mathbf{I} \\
\mathbf{0} & \mathbf{0} & \mathbf{0} & \mathbf{0} \\
\mathbf{0} & \mathbf{0} & \mathbf{0} & \mathbf{0} \\
\mathbf{0} & \mathbf{0} & \mathbf{0} & \mathbf{0}
\end{array}\right]^{\mathrm{T}} \mathbf{P}\right)\left[\begin{array}{c}
\mathbf{e}(t) \\
\mathbf{m}_{e}(t) \\
\mathbf{y}(t) \\
\mathbf{z}(t)
\end{array}\right] \\
& +\sum_{i=1}^{p} \sum_{j=1}^{p} w_{i} m_{j}\left[\begin{array}{c}
\mathbf{e}(t) \\
\mathbf{m}_{e}(t) \\
\mathbf{y}(t) \\
\mathbf{z}(t)
\end{array}\right]^{\mathrm{T}}\left(\mathbf{P}^{\mathrm{T}}\left[\begin{array}{cccc}
\mathbf{0} & \mathbf{0} & \mathbf{0} & \mathbf{0} \\
\mathbf{0} & \mathbf{0} & \mathbf{0} & \mathbf{0} \\
\mathbf{B} \mathbf{G}_{j} & \mathbf{0} & \mathbf{- I} & \mathbf{0} \\
\mathbf{A}_{i} & \mathbf{I} & \mathbf{I} & \mathbf{- I}
\end{array}\right]+\left[\begin{array}{cccc}
\mathbf{0} & \mathbf{0} & \mathbf{0} & \mathbf{0} \\
\mathbf{0} & \mathbf{0} & \mathbf{0} & \mathbf{0} \\
\mathbf{B} \mathbf{G}_{j} & \mathbf{0} & \mathbf{- I} & \mathbf{0} \\
\mathbf{A}_{i} & \mathbf{I} & \mathbf{I} & \mathbf{- I}
\end{array}\right]^{\mathrm{T}} \mathbf{P}\right)\left[\begin{array}{c}
\mathbf{e}(t) \\
\mathbf{m}_{e}(t) \\
\mathbf{y}(t) \\
\mathbf{z}(t)
\end{array}\right] \\
& =\sum_{i=1}^{p} \sum_{j=1}^{p} w_{i} m_{j}\left[\begin{array}{c}
\mathbf{e}(t) \\
\mathbf{m}_{e}(t) \\
\mathbf{y}(t) \\
\mathbf{z}(t)
\end{array}\right]^{\mathrm{T}}\left(\mathbf{P}^{\mathrm{T}}\left[\begin{array}{cccc}
\mathbf{0} & \mathbf{0} & \mathbf{0} & \mathbf{I} \\
\mathbf{0} & \mathbf{0} & \mathbf{0} & \mathbf{0} \\
\mathbf{B G}_{j} & \mathbf{0} & \mathbf{- I} & \mathbf{0} \\
\mathbf{A}_{i} & \mathbf{I} & \mathbf{I} & -\mathbf{I}
\end{array}\right]+\left[\begin{array}{cccc}
\mathbf{0} & \mathbf{0} & \mathbf{0} & \mathbf{I} \\
\mathbf{0} & \mathbf{0} & \mathbf{0} & \mathbf{0} \\
\mathbf{B} \mathbf{G}_{j} & \mathbf{0} & \mathbf{- I} & \mathbf{0} \\
\mathbf{A}_{i} & \mathbf{I} & \mathbf{I} & -\mathbf{I}
\end{array}\right]^{\mathrm{T}} \mathbf{P}\right. \\
& \left.+\left[\begin{array}{cccc}
\mathbf{P}_{1} \mathbf{U}_{i} \mathbf{P}_{1} & \mathbf{0} & \mathbf{0} & \mathbf{0} \\
\mathbf{0} & -\sigma^{2} \mathbf{P}_{1} \mathbf{U}_{i} \mathbf{P}_{1} & \mathbf{0} & \mathbf{0} \\
\mathbf{0} & \mathbf{0} & \mathbf{0} & \mathbf{0} \\
\mathbf{0} & \mathbf{0} & \mathbf{0} & \mathbf{0}
\end{array}\right]\right)\left[\begin{array}{c}
\mathbf{e}(t) \\
\mathbf{m}_{e}(t) \\
\mathbf{y}(t) \\
\mathbf{z}(t)
\end{array}\right] \\
& -\mathbf{e}(t)^{\mathrm{T}} \mathbf{P}_{1} \mathbf{U} \mathbf{P}_{1} \mathbf{e}(t)+\sigma^{2} \mathbf{m}_{e}(t)^{\mathrm{T}} \mathbf{P}_{1} \mathbf{U} \mathbf{P}_{1} \mathbf{m}_{e}(t)
\end{aligned}
$$

$$
\begin{aligned}
\dot{V}(t)= & \sum_{i=1}^{p} \sum_{j=1}^{p} w_{i} m_{j} \mathbf{v}(t)^{\mathrm{T}}\left(\left[\begin{array}{cccc}
\mathbf{0} & \mathbf{0} & \mathbf{0} & \mathbf{I} \\
\mathbf{0} & \mathbf{0} & \mathbf{0} & \mathbf{0} \\
\mathbf{B} \mathbf{G}_{j} & \mathbf{0} & -\mathbf{I} & \mathbf{0} \\
\mathbf{A}_{i} & \mathbf{I} & \mathbf{I} & -\mathbf{I}
\end{array}\right] \mathbf{X}+\mathbf{X}^{\mathrm{T}}\left[\begin{array}{cccc}
\mathbf{0} & \mathbf{0} & \mathbf{0} & \mathbf{I} \\
\mathbf{0} & \mathbf{0} & \mathbf{0} & \mathbf{0} \\
\mathbf{B} \mathbf{G}_{j} & \mathbf{0} & \mathbf{- I} & \mathbf{0} \\
\mathbf{A}_{i} & \mathbf{I} & \mathbf{I} & -\mathbf{I}
\end{array}\right]^{\mathrm{T}}\right. \\
& \left.\mathbf{X}^{\mathrm{T}}\left[\begin{array}{cccc}
\mathbf{P}_{1} \mathbf{U}_{i} \mathbf{P}_{1} & \mathbf{0} & \mathbf{0} & \mathbf{0} \\
\mathbf{0} & -\sigma^{2} \mathbf{P}_{1} \mathbf{U}_{i} \mathbf{P}_{1} & \mathbf{0} & \mathbf{0} \\
\mathbf{0} & \mathbf{0} & \mathbf{0} & \mathbf{0} \\
\mathbf{0} & \mathbf{0} & \mathbf{0} & \mathbf{0}
\end{array}\right] \mathbf{X}\right) \mathbf{v}(t)-\mathbf{e}(t)^{\mathrm{T}} \mathbf{P}_{1} \mathbf{U} \mathbf{P}_{1} \mathbf{e}(t)+\sigma^{2} \mathbf{m}_{e}(t)^{\mathrm{T}} \mathbf{P}_{1} \mathbf{U} \mathbf{P}_{1} \mathbf{m}_{e}(t) \\
= & \sum_{i=1}^{p} \sum_{j=1}^{p} w_{i} m_{j} \mathbf{v}(t)^{\mathrm{T}} \mathbf{V}_{i j} \mathbf{v}(t)-\mathbf{e}(t)^{\mathrm{T}} \mathbf{P}_{1} \mathbf{U} \mathbf{P}_{1} \mathbf{e}(t)+\sigma^{2} \mathbf{m}_{e}(t)^{\mathrm{T}} \mathbf{P}_{1} \mathbf{U} \mathbf{P}_{1} \mathbf{m}_{e}(t)
\end{aligned}
$$

$$
\mathbf{V}_{i j}=\left[\begin{array}{cccc}
\mathbf{X}_{5 i}+\mathbf{X}_{5 i}{ }^{\mathrm{T}}+\mathbf{U}_{i}-\sigma^{2} \mathbf{U}_{i} & * & * & * \\
\mathbf{X}_{6 i}^{\mathrm{T}}-\sigma^{2} \mathbf{U}_{i} & -\sigma^{2} \mathbf{U}_{i} & * & * \\
\mathbf{B N}_{j}-\mathbf{X}_{2 i}+\mathbf{X}_{7 i}{ }^{\mathrm{T}} & -\mathbf{X}_{3 i} & -\mathbf{X}_{4 i}-\mathbf{X}_{4 i}{ }^{\mathrm{T}} & * \\
\mathbf{A}_{i} \mathbf{X}_{1}+\mathbf{X}_{1}+\mathbf{X}_{2 i}-\mathbf{X}_{5 i}+\mathbf{X}_{8 i}{ }^{\mathrm{T}} & \mathbf{X}_{1}+\mathbf{X}_{3 i}-\mathbf{X}_{6 i} & \mathbf{X}_{4 i}-\mathbf{X}_{7 i} & -\mathbf{X}_{8 i}-\mathbf{X}_{8 i}{ }^{\mathrm{T}}
\end{array}\right]
$$


$m_{i}-\rho w_{i}+\gamma>0$ for all $i$ and $\mathbf{x}(t)$ where $\rho>0$ and $\gamma$ are scalars to be determined. From (18), we have

$$
\begin{aligned}
\dot{V}(t)= & \sum_{i=1}^{p} \sum_{j=1}^{p} w_{i}\left(m_{j}+\rho w_{j}-\rho w_{j}+\gamma-\gamma\right) \mathbf{v}(t)^{\mathrm{T}} \mathbf{V}_{i j} \mathbf{v}(t) \\
& -\mathbf{e}(t)^{\mathrm{T}} \mathbf{P}_{1} \mathbf{U} \mathbf{P}_{1} \mathbf{e}(t)+\sigma^{2} \mathbf{m}_{e}(t)^{\mathrm{T}} \mathbf{P}_{1} \mathbf{U} \mathbf{P}_{1} \mathbf{m}_{e}(t) \\
= & \sum_{i=1}^{p} w_{i} \mathbf{v}(t)^{\mathrm{T}}\left(\rho \mathbf{V}_{i i}-\gamma \sum_{k=1}^{p} \mathbf{V}_{i k}\right) \mathbf{v}(t) \\
& +\sum_{i=1}^{p} \sum_{j=1}^{p} w_{i}\left(m_{j}-\rho w_{j}+\gamma\right) \mathbf{v}(t)^{\mathrm{T}} \mathbf{V}_{i j} \mathbf{v}(t) \\
& +\sum_{i=1}^{p} \sum_{j=1}^{p} w_{i}\left(m_{j}-\rho w_{j}+\gamma\right) \mathbf{v}(t)^{\mathrm{T}} \boldsymbol{\Lambda}_{i} \mathbf{v}(t) \\
& -\sum_{i=1}^{p} w_{i}(1-\rho+p \gamma) \mathbf{v}(t)^{\mathrm{T}} \boldsymbol{\Lambda}_{i} \mathbf{v}(t) \\
& -\mathbf{e}(t)^{\mathrm{T}} \mathbf{P}_{1} \mathbf{U} \mathbf{P}_{1} \mathbf{e}(t)+\sigma^{2} \mathbf{m}_{e}(t)^{\mathrm{T}} \mathbf{P}_{1} \mathbf{U} \mathbf{P}_{1} \mathbf{m}_{e}(t) \\
= & \sum_{i=1}^{p} w_{i} \mathbf{v}(t)^{\mathrm{T}}\left(\rho \mathbf{V}_{i i}-(1-\rho+p \gamma) \boldsymbol{\Lambda}_{i}-\gamma \sum_{k=1}^{p} \mathbf{V}_{i k}\right) \mathbf{v}(t) \\
& +\sum_{i=1}^{p} \sum_{j=1}^{p} w_{i}\left(m_{j}-\rho w_{j}+\gamma\right) \mathbf{v}(t)^{\mathrm{T}}\left(\mathbf{V}_{i j}+\boldsymbol{\Lambda}_{i}\right) \mathbf{v}(t) \\
& -\mathbf{e}(t)^{\mathrm{T}} \mathbf{P}_{1} \mathbf{U} \mathbf{P}_{1} \mathbf{e}(t)+\sigma^{2} \mathbf{m}_{e}(t)^{\mathrm{T}} \mathbf{P}_{1} \mathbf{U} \mathbf{P}_{1} \mathbf{m}_{e}(t) \quad(19)
\end{aligned}
$$

where $\boldsymbol{\Lambda}_{i}=\boldsymbol{\Lambda}_{i}^{\mathrm{T}} \in \Re^{4 n \times 4 n}, i=1,2, \ldots, p$, are arbitrary matrices which are severed to transfer stable elements between the first two terms in the right-hand side of (19) to compensate unstable elements to produce less conservative stability analysis result. It can be seen from (19) that if $\rho \mathbf{V}_{i i}-(1-\rho+p \gamma) \boldsymbol{\Lambda}_{i}-$ $\gamma \sum_{k=1}^{p} \mathbf{V}_{i k}<0$ and $\mathbf{V}_{i j}+\Lambda_{i j}<0$ for $i, j=1,2, \ldots, p$, we have

$$
\dot{V}(t) \leq-\mathbf{e}(t)^{\mathrm{T}} \mathbf{P}_{1} \mathbf{U} \mathbf{P}_{1} \mathbf{e}(t)+\sigma^{2} \mathbf{m}_{e}(t)^{\mathrm{T}} \mathbf{P}_{1} \mathbf{U} \mathbf{P}_{1} \mathbf{m}_{e}(t)
$$

Taking integration on both sides of (20), we have (21), shown at the bottom of the page.

Based on the facts that $V(\infty) \geq 0$ and $\sigma^{2}>0$, the $H_{\infty}$ tracking performance of (21) is achieved to guarantee the tracking performance. It can be seen that a good tracking performance is ensured by a small value of $\sigma^{2}$. The stability analysis result is summarized in the following theorem.

Theorem 1: The error system of (10), formed by the response chaotic system in the form of (2), the drive chaotic system in the form of (5) and the timed-delayed fuzzy state-feedback controller of (7), satisfies the following $H_{\infty}$ tracking performance for a prescribed attenuation level $\sigma>0$

$$
\begin{aligned}
\int_{0}^{\infty} \mathbf{e}(t)^{\mathrm{T}} \mathbf{P}_{1} \mathbf{U} \mathbf{P}_{1} \mathbf{e}(t) d t \leq & V(0) \\
& +\int_{0}^{\infty} \sigma^{2} \mathbf{m}_{e}(t)^{\mathrm{T}} \mathbf{P}_{1} \mathbf{U} \mathbf{P}_{1} \mathbf{m}_{e}(t) d t
\end{aligned}
$$

if the membership functions of the time-delayed fuzzy controller are designed such that $m_{i}(\mathbf{x}(t))-\rho w_{i}(\mathbf{x}(t))+\gamma>0$ for all $i$ and $\mathbf{x}(t)$ where $\rho>0$ and $\gamma$ are scalars and there exist constant matrices $\mathbf{X}_{1}=\mathbf{X}_{1}^{\mathrm{T}} \in \Re^{n \times n}, \mathbf{X}_{k i} \in \Re^{n \times n}$, $\mathbf{N}_{j} \in \Re^{m \times n}, \mathbf{U}_{i}=\mathbf{U}_{i}^{\mathrm{T}} \in \Re^{n \times n}$, and $\boldsymbol{\Lambda}_{i}=\boldsymbol{\Lambda}_{i}^{\mathrm{T}} \in \Re^{4 n \times 4 n}$ such that the following LMIs hold.

$\mathbf{X}_{1}>0 ; \mathbf{U}_{i}>0, i=1,2, \ldots, p$;

$\rho \mathbf{V}_{i i}-(1-\rho+p \gamma) \boldsymbol{\Lambda}_{i}-\gamma \sum_{k=1}^{p} \mathbf{V}_{i k}<0, i=1,2, \ldots, p$;

$\mathbf{V}_{i j}+\boldsymbol{\Lambda}_{i}<0, i, j=1,2, \ldots, p$, and the feedback gains are defined as $\mathbf{G}_{i}=\mathbf{N}_{i} \mathbf{X}_{1}{ }^{-1}, i=1,2, \ldots, p$.

Remark 1: The above analysis is valid if $\mathbf{X}$ is invertible. Referring to Theorem 1, if there exists a solution to the stability conditions in Theorem 1, it implies that $\mathbf{X}_{1}>0, \mathbf{X}_{4 i}+\mathbf{X}_{4 i}{ }^{\mathrm{T}}>$ 0 and $\mathbf{X}_{8 i}+\mathbf{X}_{8 i}{ }^{\mathrm{T}}>0$ for all $i$. These are sufficient conditions for $\mathbf{X}$ to be a nonsingular matrix to ensure that $\mathbf{X}$ is invertible.

Remark 2: Referring to Theorem 1, it can be seen that the stability conditions do not relate to the information of the time delay. Hence, the error system is guaranteed to be stable for any value of time delay.

\section{Simulation Examples}

Three examples are given in this section to illustrate the effectiveness of the proposed approach.

\section{A. Example 1}

Two Rössler systems subject to parameter uncertainties are employed as the response and drive chaotic systems, respectively. The proposed time-delayed fuzzy controller is employed to realize the synchronization.

Step 1) The dynamics of the response Rössler's system with input term are described as follows:

$$
\dot{\mathbf{x}}(t)=\mathbf{A}(\mathbf{x}(t)) \mathbf{x}(t)+\mathbf{B} u(t)
$$

where

$$
\mathbf{x}(t)=\left[\begin{array}{l}
x_{1}(t) \\
x_{2}(t) \\
x_{3}(t)
\end{array}\right]
$$

$$
\begin{aligned}
\int_{0}^{\infty} \dot{V}(t) d t & \leq \int_{0}^{\infty}\left(-\mathbf{e}(t)^{\mathrm{T}} \mathbf{P}_{1} \mathbf{U} \mathbf{P}_{1} \mathbf{e}(t)+\sigma^{2} \mathbf{m}_{e}(t)^{\mathrm{T}} \mathbf{P}_{1} \mathbf{U} \mathbf{P}_{1} \mathbf{m}_{e}(t)\right) d t \\
V(\infty)-V(0) & \leq \int_{0}^{\infty}\left(-\mathbf{e}(t)^{\mathrm{T}} \mathbf{P}_{1} \mathbf{U} \mathbf{P}_{1} \mathbf{e}(t)+\sigma^{2} \mathbf{m}_{e}(t)^{\mathrm{T}} \mathbf{P}_{1} \mathbf{U} \mathbf{P}_{1} \mathbf{m}_{e}(t)\right) d t \\
\int_{0}^{\infty} \mathbf{e}(t)^{\mathrm{T}} \mathbf{P}_{1} \mathbf{U} \mathbf{P}_{1} \mathbf{e}(t) d t & \leq V(0)+\int_{0}^{\infty} \sigma^{2} \mathbf{m}_{e}(t)^{\mathrm{T}} \mathbf{P}_{1} \mathbf{U} \mathbf{P}_{1} \mathbf{m}_{e}(t) d t
\end{aligned}
$$




$$
\begin{aligned}
\mathbf{A}(\mathbf{x}(t)) & =\left[\begin{array}{ccc}
0 & -1 & -1 \\
1 & a & 0 \\
b & 0 & -\left(c(t)-x_{1}(t)\right)
\end{array}\right] \\
\mathbf{B} & =\left[\begin{array}{l}
0 \\
0 \\
1
\end{array}\right]
\end{aligned}
$$

and $a=0.34, b=0.4$

$c(t)=\frac{c_{\max }+c_{\min }}{2}+\frac{c_{\max }-c_{\min }}{2} \sin (t) \in\left[c_{\min } c_{\max }\right]>0$

is the uncertain parameter, $c_{\min }=4.5$, and $c_{\max }=7.7$. It is assumed that $x_{1}(t) \in$ $\left[\begin{array}{cc}c_{\max }-d & c_{\min }+d\end{array}\right]$ and $d=25$. The response Rössler system can be exactly represented by a fuzzy model with the following fuzzy rules [3]-[5]:

Rule $i$ :IF $x_{1}(t)$ is $\mathrm{M}^{i}$

THEN $\dot{\mathbf{x}}(t)=\mathbf{A}_{i} \mathbf{x}(t)+\mathbf{B} u(t), \quad i=1,2$.

The inferred response Rössler system is defined as

$\dot{\mathbf{x}}(t)=\sum_{i=1}^{2} w_{i}\left(x_{1}(t)\right)\left(\mathbf{A}_{i} \mathbf{x}(t)+\mathbf{B} u(t)\right)$

where

$$
\begin{aligned}
& \mathbf{A}_{1}=\left[\begin{array}{ccc}
0 & -1 & -1 \\
1 & a & 0 \\
b & 0 & -d
\end{array}\right] \\
& \mathbf{A}_{2}=\left[\begin{array}{ccc}
0 & -1 & -1 \\
1 & a & 0 \\
b & 0 & d
\end{array}\right]
\end{aligned}
$$

and $w_{1}\left(x_{1}(t)\right)=\mu_{\mathrm{M}^{1}}\left(x_{1}(t)\right)=$ $1 / 2\left(1+\left(\left(c(t)-x_{1}(t)\right) / d\right)\right)$ and $w_{2}\left(x_{1}(t)\right)=$ $\mu_{\mathrm{M}^{2}}\left(x_{1}(t)\right)=1-\mu_{\mathrm{M}^{1}}\left(x_{1}(t)\right)$. It can be seen that the uncertain parameter $c(t)$ makes the grades of membership function uncertain in value. Consequently, the proposed approaches in [3]-[6], [12]-[16] for uncertainty-free chaotic systems cannot be applied.

Step 2) The dynamics of the drive Rössler's system subject to parameter uncertainties are given as follows:

$$
\dot{\hat{\mathbf{x}}}(t)=\hat{\mathbf{A}}(\hat{\mathbf{x}}(t)) \hat{\mathbf{x}}(t)
$$

where

$$
\begin{aligned}
\hat{\mathbf{x}}(t) & =\left[\begin{array}{l}
\hat{x}_{1}(t) \\
\hat{x}_{2}(t) \\
\hat{x}_{3}(t)
\end{array}\right] \\
\hat{\mathbf{A}}(\hat{\mathbf{x}}(t)) & =\left[\begin{array}{ccc}
0 & -1 & -1 \\
1 & \hat{a} & 0 \\
\hat{b} & 0 & -\left(\hat{c}(t)-\hat{x}_{1}(t)\right)
\end{array}\right]
\end{aligned}
$$

where $\hat{a}=0.34, \hat{b}=0.4, \hat{c}(t)=\left(\hat{c}_{2}+\hat{c}_{1}\right) / 2+$ $\left(\left(\hat{c}_{2}-\hat{c}_{1}\right) / 2\right) \cos (t)$ is regarded as the parameter uncertainty, $\hat{c}_{1}=4.5$ and $\hat{c}_{1}=7.7$.

Step 3) The fuzzy state-feedback controller of (7) is employed to handle the synchronization problem
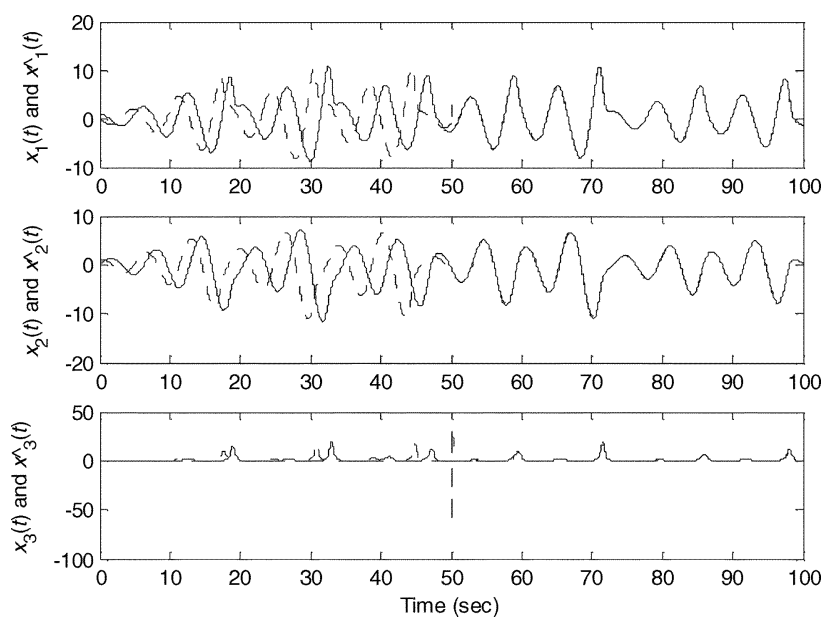

Fig. 2. System state responses of the response (dotted lines) and drive (solid lines) Rössler systems with $u(t)=0$ for $0 \leq t<50 \mathrm{~s}$ and the proposed fuzzy controller applied for $t \geq 50 \mathrm{~s}$ with $\tau_{d}(t)=0.01(1+(1+\sin (t)) / 4)$.

using the time-delayed system state information of the drive system. The fuzzy rules are designed as follows:

Rule $j$ :IF $x_{1}(t)$ is $\mathrm{N}_{1}^{j}$

$$
\text { THEN } u(t)=\mathbf{G}_{j}\left(\mathbf{x}(t)-\hat{\mathbf{x}}\left(t-\tau_{d}(t)\right)\right), \quad j=1,2
$$

The inferred fuzzy controller is defined as

$$
u(t)=\sum_{j=1}^{2} m_{j}\left(x_{1}(t)\right) \mathbf{G}_{j}\left(\mathbf{x}(t)-\hat{\mathbf{x}}\left(t-\tau_{d}(t)\right)\right)
$$

where the membership functions are designed as $m_{1}\left(x_{1}(t)\right)=\mu_{\mathrm{N}_{1}^{1}}\left(x_{1}(t)\right)=-0.0179\left(x_{1}(t)-\right.$ $29.5)+0.0804$ and $m_{2}\left(x_{1}(t)\right)=1-\mu_{\mathrm{N}_{1}^{2}}\left(x_{1}(t)\right)=$ $1-m_{1}\left(x_{1}(t)\right)$ for $x_{1}(t) \in\left[c_{\max }-d \quad c_{\min }+d\right]$. It can be shown that the condition of $m_{j}\left(x_{1}(t)\right)-$ $\rho w_{j}\left(x_{1}(t)\right)+\gamma>0$ for all $j$ and $x_{1}(t)$, with $\rho=$ 0.91 and $\gamma=0$, is satisfied. By solving the solution to the stability conditions in Theorem 1 using MATLAB LMI toolbox, with $\sigma=0.1$, we have $\mathbf{G}_{1}=\left[\begin{array}{lll}1202.0599 & 8403.8542 & -38.3676\end{array}\right]$ and $\mathbf{G}_{2}=\left[\begin{array}{lll}657.4397 & 4602.8992 & -51.5845\end{array}\right]$.

Fig. 2 shows the system state responses of the response and drive chaotic systems under the initial conditions of $\hat{\mathbf{x}}(t)=$ $\left[\begin{array}{lll}0 & 1 & 0\end{array}\right]^{\mathrm{T}}$ and $\mathbf{x}(t)=\left[\begin{array}{lll}1 & 0 & 0\end{array}\right]^{\mathrm{T}}$ for $t \geq 0$. In this simulation, $u(t)=0$ is employed for $0 \leq t<50 \mathrm{~s}$ and the fuzzy state-feedback controller is applied for $t \geq 50 \mathrm{~s}$ with $\tau_{d}(t)=$ $0.01(1+(1+\sin (t)) / 4)$. Fig. 3 shows the tracking error between the response and drive systems. Referring to these figures, it can be seen that the proposed fuzzy controller, which is applied for $t \geq 50 \mathrm{~s}$, is able to drive the system states of the uncertain response Rössler's system to follow those of the uncertain drive Rössler's system with a sufficiently small tracking error. The simulation is repeated for $\tau_{d}(t)=0.1(1+(1+\sin (t)) / 4)$ and $\tau_{d}(t)=1+(1+\sin (t)) / 4$, respectively . Figs. 4-7 show the system responses and the tracking error between the response and drive systems, respectively. Referring to these fig- 

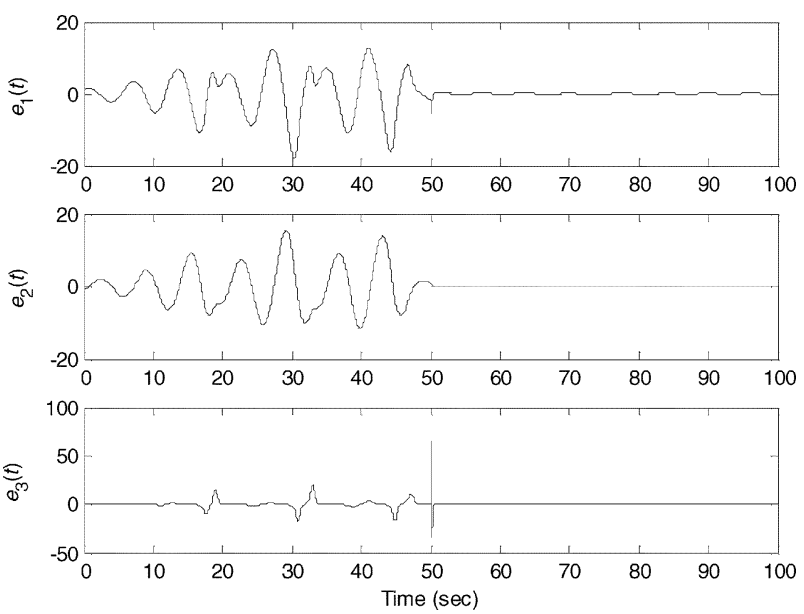

Fig. 3. Tracking error of the response Rössler system with $u(t)=0$ for $0 \leq$ $t<50 \mathrm{~s}$ and the proposed fuzzy controller applied for $t \geq 50 \mathrm{~s}$ with $\tau_{d}(t)=$ $0.01(1+(1+\sin (t)) / 4)$.
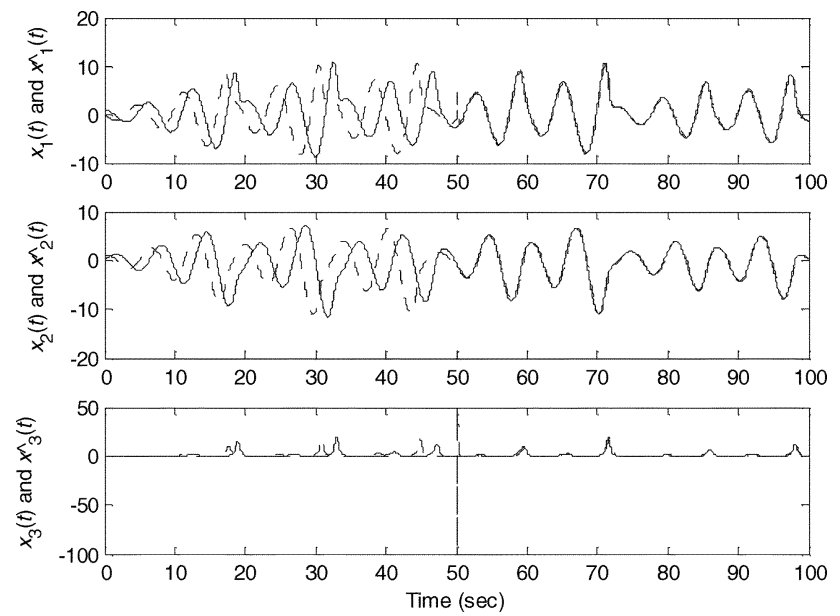

Fig. 4. System state responses of the response (dotted lines) and drive (solid lines) Rössler systems with $u(t)=0$ for $0 \leq t<50 \mathrm{~s}$ and the proposed fuzzy controller applied for $t \geq 50 \mathrm{~s}$ with $\tau_{d}(t)=0.1(1+(1+\sin (t)) / 4)$
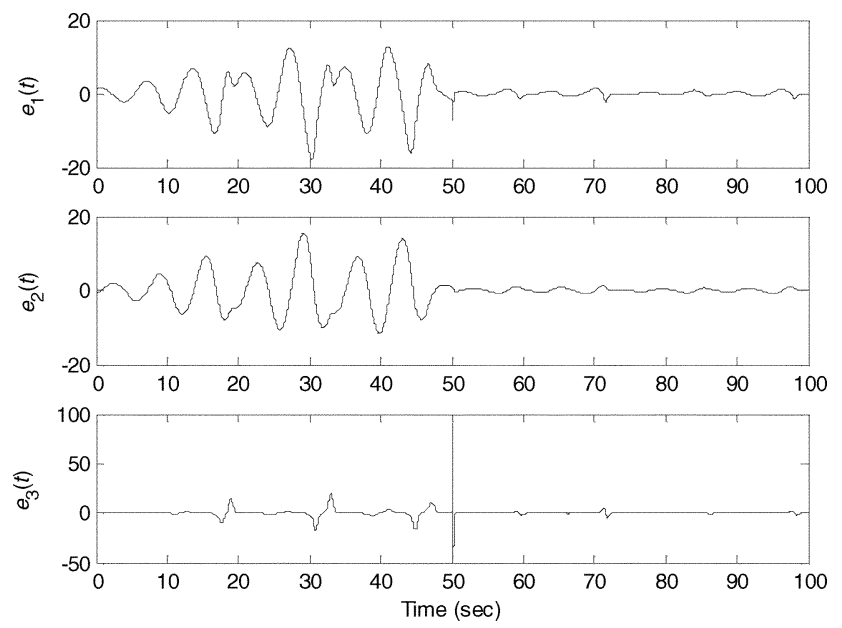

Fig. 5. Tracking error of the response Rössler system with $u(t)=0$ for $0 \leq$ $t<50 \mathrm{~s}$ and the proposed fuzzy controller applied for $t \geq 50 \mathrm{~s}$ with $\tau_{d}(t)=$ $0.1(1+(1+\sin (t)) / 4)$.

ures, it can be seen that the tracking error cannot be kept small for $\tau_{d}(t)=1+(1+\sin (t)) / 4$ but bounded when the value of time delay is sufficiently large.
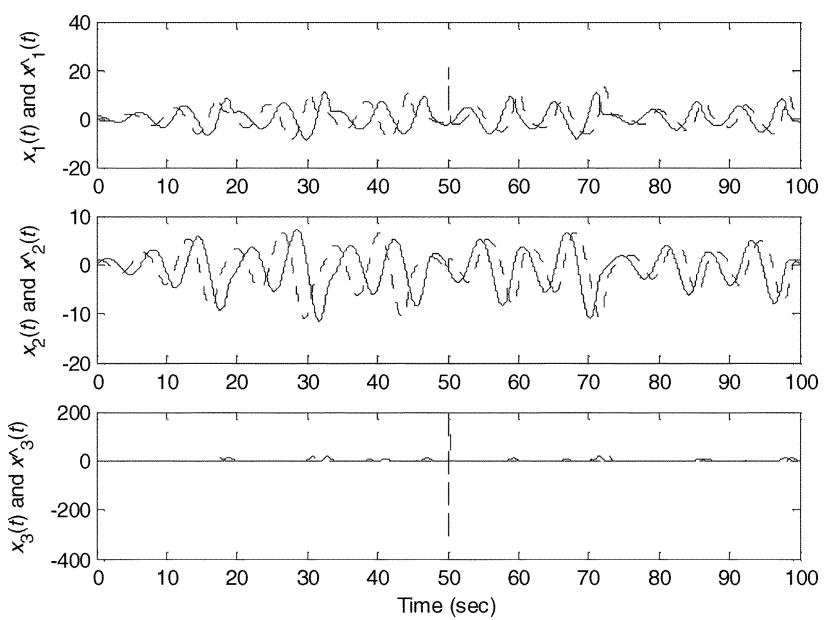

Fig. 6. System state responses of the response (dotted lines) and drive (solid lines) Rössler systems with $u(t)=0$ for $0 \leq t<50 \mathrm{~s}$ and the proposed fuzzy controller applied for $t \geq 50 \mathrm{~s}$ with $\tau_{d}(t)=1+(1+\sin (t)) / 4$.

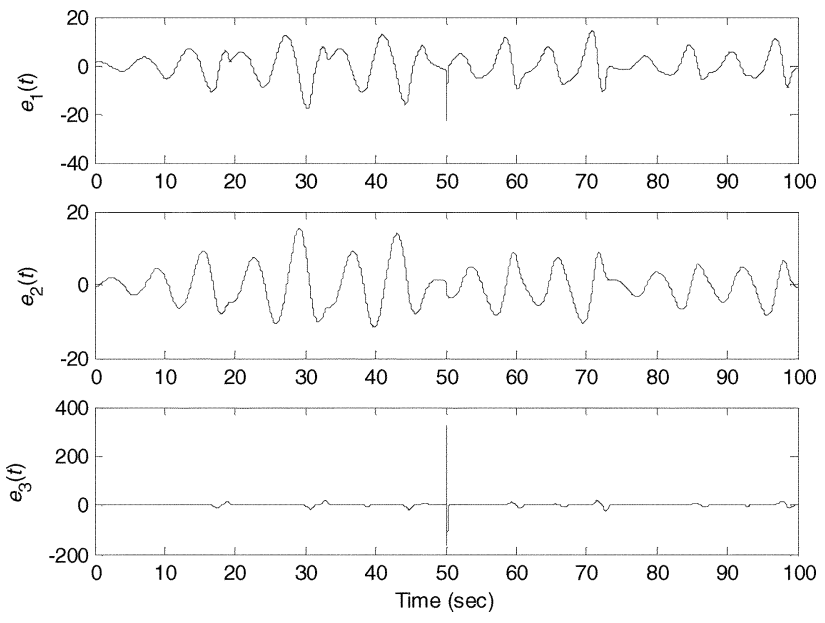

Fig. 7. Tracking error of the response Rössler system with $u(t)=0$ for $0 \leq$ $t<50 \mathrm{~s}$ and the proposed fuzzy controller applied for $t \geq 50 \mathrm{~s}$ with $\tau_{d}(t)=$ $1+(1+\sin (t)) / 4$.

In this example, the proposed fuzzy controller is able to synchronize both the response and drive chaotic systems subject to parameter uncertainties and time-varying delay. However, the theories developed in [3]-[6], [12]-[16] for uncertainty-free chaotic systems with constant time delay cannot be applied to handle the synchronization problems considered. Compared with the fuzzy adaptive controller in [8]-[11] for chaotic systems, the proposed fuzzy controller offers lower computational demand and structural complexity. Moreover, the time delay is not considered in [8]-[11].

\section{B. Example 2}

Two Chen's systems [16] are considered as the drive and responses systems. In this example, the proposed control scheme is compared with that in [16] of which no time delay and parameter uncertainties are considered. It can be seen that the proposed fuzzy controllers offer simpler structure and better performance.

The Chen's system can be exactly represented by the fuzzy model with two fuzzy rules [16] in the form of (23). It should be noted that the drive Chen's system does not have the control 
input of $\mathbf{u}(t)$. The inferred Chen's system [16] is in the form of (24) with

$$
\begin{aligned}
\mathbf{A}_{1}= & {\left[\begin{array}{ccc}
-a & a & 0 \\
c-a & c & -d \\
0 & d & -b
\end{array}\right] } \\
\mathbf{A}_{2}= & {\left[\begin{array}{ccc}
-a & a & 0 \\
c-a & c & d \\
0 & -d & -b
\end{array}\right] } \\
\mathbf{B} & =\left[\begin{array}{lll}
1 & 0 & 0 \\
0 & 1 & 0 \\
0 & 0 & 1
\end{array}\right]
\end{aligned}
$$

where $a=35, b=3, c=28$ and $d=30$. The membership functions for the drive and response systems are defined as $\hat{w}_{1}\left(\hat{x}_{1}(t)\right)=\mu_{\mathrm{M}^{1}}\left(\hat{x}_{1}(t)\right)=(1 / 2)\left(1+\left(\hat{x}_{1}(t) / d\right)\right)$ and $\hat{w}_{2}\left(\hat{x}_{1}(t)\right)=\mu_{\mathrm{M}^{2}}\left(\hat{x}_{1}(t)\right)=1-\mu_{\mathrm{M}^{1}}\left(\hat{x}_{1}(t)\right)$, $w_{1}\left(x_{1}(t)\right)=\mu_{\mathrm{M}^{1}}\left(x_{1}(t)\right)=(1 / 2)\left(1+\left(x_{1}(t) / d\right)\right)$ and $w_{2}\left(x_{1}(t)\right)=\mu_{\mathrm{M}^{2}}\left(x_{1}(t)\right)=1-\mu_{\mathrm{M}^{1}}\left(x_{1}(t)\right)$, respectively. It is assumed that both the drive and response chaotic systems work in the operating domain of $\hat{x}_{1}(t) \in\left[\begin{array}{ll}-d & d\end{array}\right]$ and $x_{1}(t) \in\left[\begin{array}{ll}-d & d\end{array}\right]$. Consequently, the fuzzy model for the drive and response Chen's systems are defined, respectively, as follows:

$$
\begin{aligned}
\dot{\hat{\mathbf{x}}}(t) & =\sum_{i=1}^{2} \hat{w}_{i}\left(\hat{x}_{1}(t)\right) \mathbf{A}_{i} \hat{\mathbf{x}}(t) \\
\dot{\mathbf{x}}(t) & =\sum_{i=1}^{2} w_{i}\left(x_{1}(t)\right)\left(\mathbf{A}_{i} \mathbf{x}(t)+\mathbf{B u}(t)\right) .
\end{aligned}
$$

Referring to [16], the fuzzy controller is defined as follows:

$$
u(t)=\sum_{j=1}^{2} \hat{w}_{j}\left(\hat{x}_{1}(t)\right) \mathbf{F}_{j} \hat{\mathbf{x}}(t)-\sum_{j=1}^{2} w_{j}\left(x_{1}(t)\right) \mathbf{F}_{j} \mathbf{x}(t)
$$

where

$$
\begin{aligned}
& \mathbf{F}_{1}=\left[\begin{array}{ccc}
-34.5000 & 13.8737 & -0.1086 \\
14.1263 & 28.5000 & -31.1732 \\
0.1086 & 31.1732 & -2.5000
\end{array}\right] \\
& \mathbf{F}_{1}=\left[\begin{array}{ccc}
-34.5000 & 13.8737 & -0.1086 \\
14.1263 & 28.5000 & 28.8268 \\
0.1086 & 28.8268 & -2.5000
\end{array}\right] .
\end{aligned}
$$

For comparison purpose, the proposed fuzzy controller with two rules is employed to realize the synchronization. The rule of the proposed fuzzy controller is in the form of (26). As input time delay in [16] is not considered, for fairness of comparison, we set $\tau_{d}(t)=0$ for the proposed fuzzy controller which is defined as follows:

$$
\mathbf{u}(t)=\sum_{j=1}^{2} m_{j}\left(x_{1}(t)\right) \mathbf{G}_{j}(\mathbf{x}(t)-\hat{\mathbf{x}}(t)) .
$$

As the membership functions of the fuzzy model of the response system do not have parameter uncertainties, the fuzzy controller
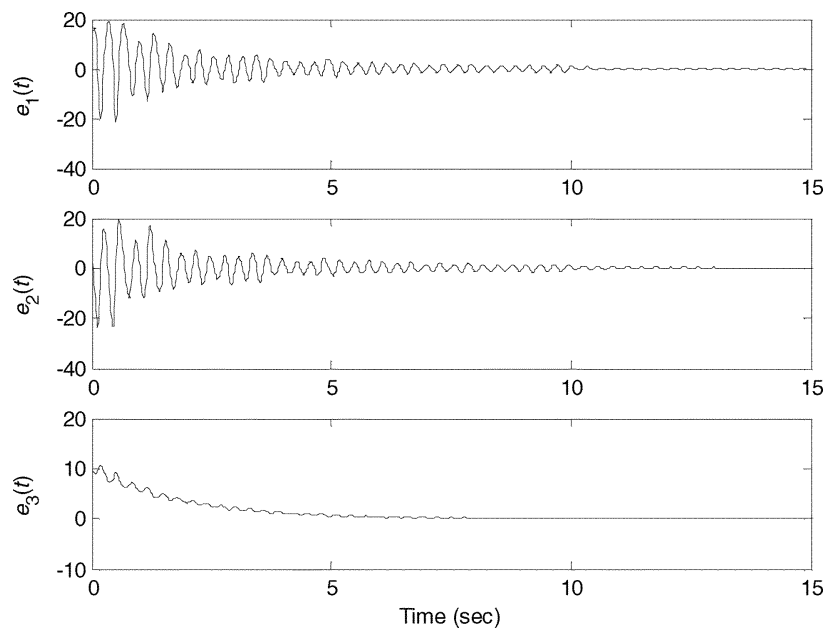

Fig. 8. Tracking error of the response Chen's system with the fuzzy controller in [16].
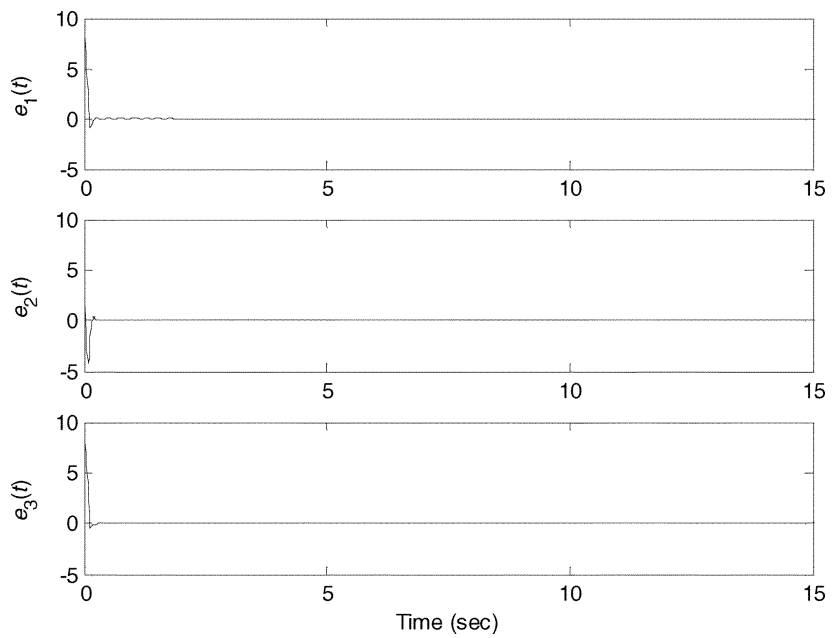

Fig. 9. Tracking error of the response Chen's system with the proposed fuzzy controller.

of (31) share the same membership functions as those of the response system, i.e, $m_{i}(\mathbf{x}(t))=w_{i}(\mathbf{x}(t)), i=1$, 2. Under such a situation, we have $\rho=1$ and $\gamma=0$ such that the membership function condition of $m_{i}(\mathbf{x}(t))-\rho w_{i}(\mathbf{x}(t))+\gamma>0$ satisfies. It should be noted that when $\rho=1$ and $\gamma=0$, the stability condition of $\mathbf{V}_{i j}+\Lambda_{i}<0$ can be removed from Theorem 1 . By solving the solution to the stability conditions in Theorem 1 using MATLAB LMI toolbox, with $\rho=1$ and $\gamma=0$ and $\sigma=0.1$, we have

$$
\begin{aligned}
& \mathbf{G}_{1}=\left[\begin{array}{ccc}
15.7209 & -17.2495 & -0.0033 \\
-10.7505 & -47.2791 & 4.6147 \\
0.0033 & -4.6147 & -16.2791
\end{array}\right] \\
& \mathbf{G}_{2}=\left[\begin{array}{ccc}
15.7209 & -17.2318 & -0.0815 \\
-10.7682 & -47.2791 & -4.7057 \\
0.0815 & 4.7057 & -16.2791
\end{array}\right] .
\end{aligned}
$$

Figs. 8 and 9 show the tracking error between the response and drive Chen's systems with the fuzzy controller of (30) and the proposed fuzzy controller of (31) for initial system states 
of $\hat{\mathbf{x}}(0)=\left[\begin{array}{lll}10 & 10 & 10\end{array}\right]^{\mathrm{T}}$ and $\mathbf{x}(0)=\left[\begin{array}{lll}0 & 0 & 0\end{array}\right]^{\mathrm{T}}$. It can be seen from the figures that the proposed fuzzy controller performs better in terms of shorter converge time. The converge time for the fuzzy controller of (30) in [16] is about $10.5 \mathrm{~s}, 13 \mathrm{~s}$ and $8 \mathrm{~s}$ for $e_{1}(t), e_{2}(t)$ and $e_{3}(t)$, respectively. While for the propose fuzzy controller of (31), the converge time is about $0.3 \mathrm{~s}$ for all $e_{1}(t), e_{2}(t)$ and $e_{3}(t)$. Furthermore, although both fuzzy controllers of (30) and (31) are able to synchronize the driven and response systems, the proposed fuzzy controller offers a simpler structure as only the membership functions of the response system are used.

\section{Example 3}

Two Lorenz systems [10] are considered as the drive and response systems. The drive Lorenz is subject to parameter uncertainties. The fuzzy adaptive control scheme in [10] is employed to synchronize the Lorenz systems and is compared to the proposed fuzzy control approach. In [10], the following rule is employed to describe the system dynamics of the Lorenz system subject to parameter uncertainties

$$
\begin{aligned}
\text { Rule } i & : \operatorname{IF} \hat{x}_{1}(t) \text { is } \mathrm{M}^{i} \\
\text { THEN } \dot{\hat{\mathbf{x}}}(t) & =\left(\hat{\mathbf{A}}_{i}+\mathbf{A}_{u i}\right) \hat{\mathbf{x}}(t), \quad i=1,2 .
\end{aligned}
$$

where

$$
\begin{aligned}
\hat{\mathbf{A}}_{1} & =\left[\begin{array}{ccc}
0 & 0 & 0 \\
0 & -1 & -d \\
0 & d & 0
\end{array}\right] \\
\hat{\mathbf{A}}_{2} & =\left[\begin{array}{ccc}
0 & 0 & 0 \\
0 & -1 & d \\
0 & -d & 0
\end{array}\right] \\
\mathbf{A}_{u 1} & =\mathbf{A}_{u 2} \\
& =\left[\begin{array}{ccc}
-\alpha & \alpha & 0 \\
\beta & 0 & 0 \\
0 & 0 & -\gamma
\end{array}\right]
\end{aligned}
$$

and $\alpha=10, \beta=28$ and $\gamma=8 / 3$. The parameters of $\alpha, \beta$ and $\gamma$ are assumed to be unknown in this example [10]. It is assumed that $\hat{x}_{1}(t) \in[-d, d]$ with $d=20$. The inferred Lorenz system is defined as follows:

$$
\dot{\hat{\mathbf{x}}}(t)=\sum_{i=1}^{p} w_{i}\left(\hat{x}_{1}(t)\right)\left(\hat{\mathbf{A}}_{i}+\mathbf{A}_{u i}\right) \hat{\mathbf{x}}(t)
$$

where the membership function are defined as $w_{1}\left(\hat{x}_{1}(t)\right)=$ $\mu_{\mathrm{M}^{1}}\left(\hat{x}_{1}(t)\right)=(1 / 2)\left(1+\left(\hat{x}_{1}(t) / d\right)\right)$ and $w_{2}\left(\hat{x}_{1}(t)\right)=$ $\mu_{\mathrm{M}^{2}}\left(\hat{x}_{1}(t)\right)=1-\mu_{\mathrm{M}^{1}}\left(\hat{x}_{1}(t)\right)$. The fuzzy response system in [10] is described by the follow rules.

Rule $i: \operatorname{IF} \hat{x}_{1}(t) \mathrm{M}^{i}$

$$
\text { THEN } \dot{\mathbf{x}}(t)=\left(\hat{\mathbf{A}}_{i}+\hat{\mathbf{A}}_{u i}\right) \hat{\mathbf{x}}(t)+\mathbf{B u}(t), \quad i=1,2 .
$$
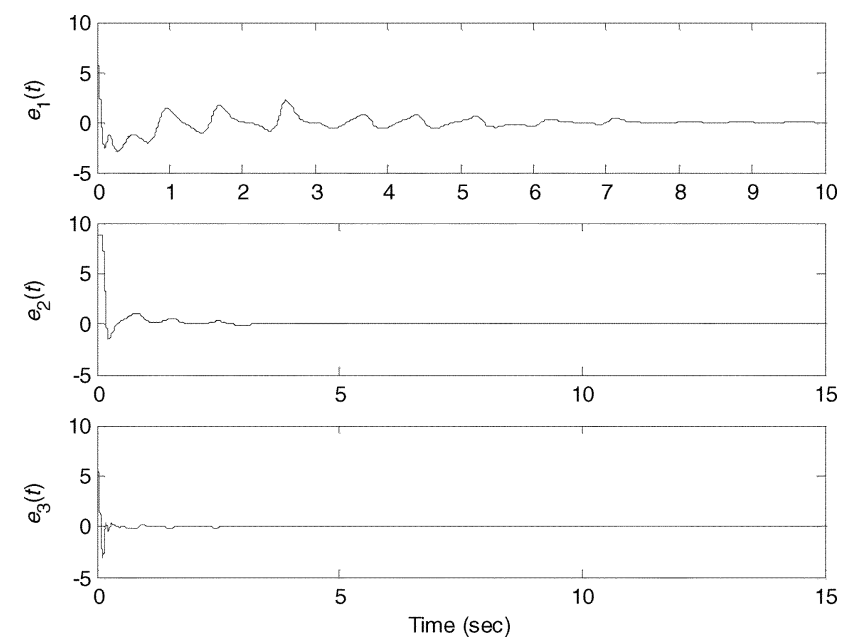

Fig. 10. Tracking error of the response Lorenz system with the fuzzy adaptive controller in [10].

where

$$
\begin{aligned}
\mathbf{B} & =\left[\begin{array}{lll}
1 & 0 & 0 \\
0 & 1 & 0 \\
0 & 0 & 1
\end{array}\right] \\
\hat{\mathbf{A}}_{u 1} & =\left[\begin{array}{ccc}
-\hat{\alpha}_{11} & \hat{\alpha}_{12} & 0 \\
\hat{\beta}_{1} & 0 & 0 \\
0 & 0 & -\hat{\gamma}_{1}
\end{array}\right] \\
\hat{\mathbf{A}}_{u 2} & =\left[\begin{array}{ccc}
-\hat{\alpha}_{21} & \hat{\alpha}_{22} & 0 \\
\hat{\beta}_{2} & 0 & 0 \\
0 & 0 & -\hat{\gamma}_{2}
\end{array}\right]
\end{aligned}
$$

are the estimates of $\mathbf{A}_{u 1}$ and $\mathbf{A}_{u 2}$, respectively. According to [10], the adaptive law is defined as

$$
\dot{\hat{\mathbf{A}}}_{u i}=\sigma_{A_{u i}} \hat{w}_{i}\left(\hat{x}_{1}(t)\right)(\hat{\mathbf{x}}(t)-\mathbf{x}(t)) \mathbf{x}(t)^{\mathrm{T}}, \quad i=1,2
$$

where $\sigma_{A_{u 1}}=2$ and $\sigma_{A_{u 2}}=2.5$ are constant adaptation gains. Given in [10], the rule of the fuzzy adaptive controller is of the following form:

$$
\begin{aligned}
\text { Rule } i & : \operatorname{IF} \hat{x}_{1}(t) \text { is } \mathrm{M}^{j} \\
\text { THEN } \mathbf{u}(t) & =\left(\hat{\mathbf{A}}_{u j}+\mathbf{K}_{j}\right) \hat{\mathbf{x}}(t), \quad i=1,2 .
\end{aligned}
$$

The inferred control law is defined as follows:

$$
\mathbf{u}(t)=\sum_{j=1}^{2} \hat{w}_{j}\left(\hat{x}_{1}(t)\right)\left(\hat{\mathbf{A}}_{u j}+\mathbf{K}_{j}\right) \hat{\mathbf{x}}(t)
$$

where

$$
\begin{aligned}
\mathbf{K}_{1} & =\left[\begin{array}{ccc}
19.3222 & 0 & 0 \\
0 & 19.9970 & 0 \\
0 & 0 & 19.7788
\end{array}\right] \\
\mathbf{K}_{2} & =\left[\begin{array}{ccc}
28.9833 & 0 & 0 \\
0 & 29.9955 & 0 \\
0 & 0 & 29.6682
\end{array}\right] .
\end{aligned}
$$

Fig. 10 shows the tracking error between the response and drive Lorenz systems with the fuzzy adaptive controller of (37). 
The initial system states are set to be $\hat{\mathbf{x}}(0)=\left[\begin{array}{lll}10 & 10 & 10\end{array}\right]^{\mathrm{T}}$ and $\mathbf{x}(0)=\left[\begin{array}{lll}0 & 0 & 0\end{array}\right]^{\mathrm{T}}$, and the initial values of $\hat{\alpha}_{11}, \hat{\alpha}_{12}$, $\hat{\alpha}_{21}, \hat{\alpha}_{22}, \hat{\beta}_{1}, \hat{\beta}_{2}, \hat{\gamma}_{1}$ and $\hat{\gamma}_{2}$ are all set to be zero. Referring to this figure, it can be seen that the fuzzy adaptive controller in $[10]$ is able to synchronize the drive and response systems. The converge time for $e_{1}(t), e_{2}(t)$ and $e_{3}(t)$ is about 7.5, 3.5, and 3 $\mathrm{s}$, respectively.

For comparison purpose, the proposed fuzzy controller is employed to realize the synchronization. To design the proposed fuzzy controller, a fuzzy model with two rules is employed to exactly describe the system behavior of the response Lorenz system. The fuzzy rule is in the form of (23) and the inferred Lorenz system is defined as in (24) with

$$
\begin{aligned}
\mathbf{A}_{1} & =\left[\begin{array}{ccc}
-a & a & 0 \\
b & -1 & -d \\
0 & d & -c
\end{array}\right] \\
\mathbf{A}_{2} & =\left[\begin{array}{ccc}
-a & a & 0 \\
b & -1 & d \\
0 & -d & -c
\end{array}\right]
\end{aligned}
$$

where $a=12, b=30$ and $c=3$. As the parameter values of the drive Lorenz system are assumed to be unknown, the parameter values, $a, b$ and $c$, of the response Lorenz system are chosen arbitrarily. The membership functions of the fuzzy model are defined as $w_{1}\left(x_{1}(t)\right)=\mu_{\mathrm{M}^{1}}\left(x_{1}(t)\right)=(1 / 2)\left(1+\left(x_{1}(t) / d\right)\right)$ and $w_{2}\left(x_{1}(t)\right)=\mu_{\mathrm{M}^{2}}\left(x_{1}(t)\right)=1-\mu_{\mathrm{M}^{1}}\left(x_{1}(t)\right)$. As no input time delay is considered in [10], for fairness of comparison, we set $\tau_{d}(t)=0$ for the proposed fuzzy controller in the form of (31). The fuzzy controller shares the same membership functions of the fuzzy model. As a result, we have $\rho=1$ and $\gamma=0$ such that the membership function condition of $m_{i}(\mathbf{x}(t))-\rho w_{i}(\mathbf{x}(t))+$ $\gamma>0$ satisfies. By solving the solution to the stability conditions in Theorem 1 using MATLAB LMI toolbox, with $\rho=1$ and $\gamma=0$ and $\sigma=0.002$, we have

$$
\begin{aligned}
\mathbf{G}_{1} & =\left[\begin{array}{ccc}
-951.1174 & -19.6154 & 0 \\
-22.3846 & -962.1174 & 3.0796 \\
0 & -3.0796 & -960.1174
\end{array}\right] \\
\mathbf{G}_{2} & =\left[\begin{array}{ccc}
-951.1174 & -19.6154 & 0 \\
-22.3846 & -962.1174 & -3.0769 \\
0 & 3.0796 & -960.1174
\end{array}\right] .
\end{aligned}
$$

Fig. 11 shows the tracking error between the response and drive Lorenz systems with the proposed fuzzy controller in the form of (31). The initial system states are set to be $\hat{\mathbf{x}}(0)=$ $\left[\begin{array}{lll}10 & 10 & 10\end{array}\right]^{\mathrm{T}}$ and $\mathbf{x}(0)=\left[\begin{array}{lll}0 & 0 & 0\end{array}\right]^{\mathrm{T}}$. It can be seen that fuzzy controller is able to synchronize the drive and response Lorenz systems with the converge time of about $0.006 \mathrm{~s}$ for all $e_{1}(t), e_{2}(t)$ and $e_{3}(t)$.

Referring to Figs. 10 and 11, it can be seen that the proposed fuzzy controller performs better in terms of shorter converge time. Furthermore, compared to the fuzzy adaptive scheme in [10], it can be seen that the proposed fuzzy control scheme offers a simpler approach to realize the synchronization in terms of lower structural complexity and computational demand for the response system and fuzzy controller.
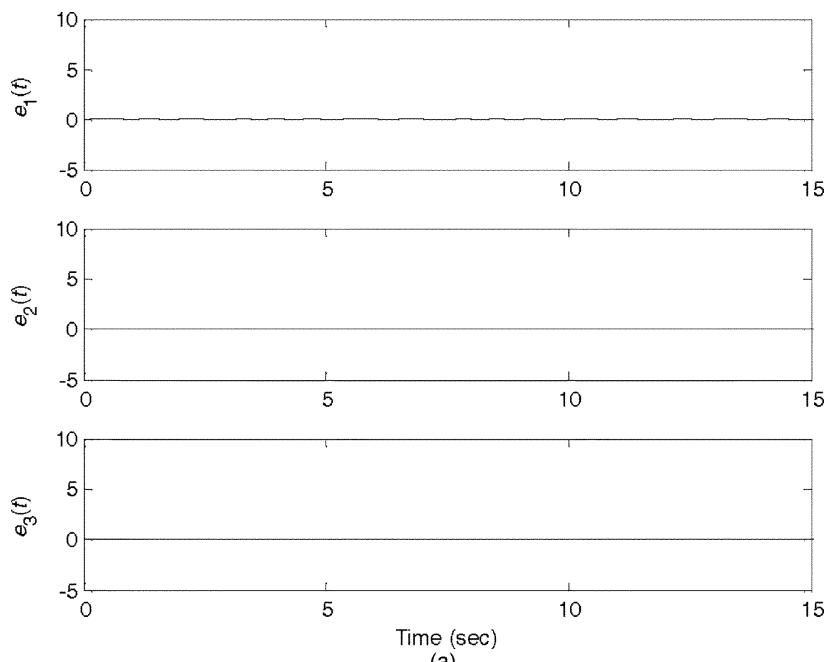

(a)
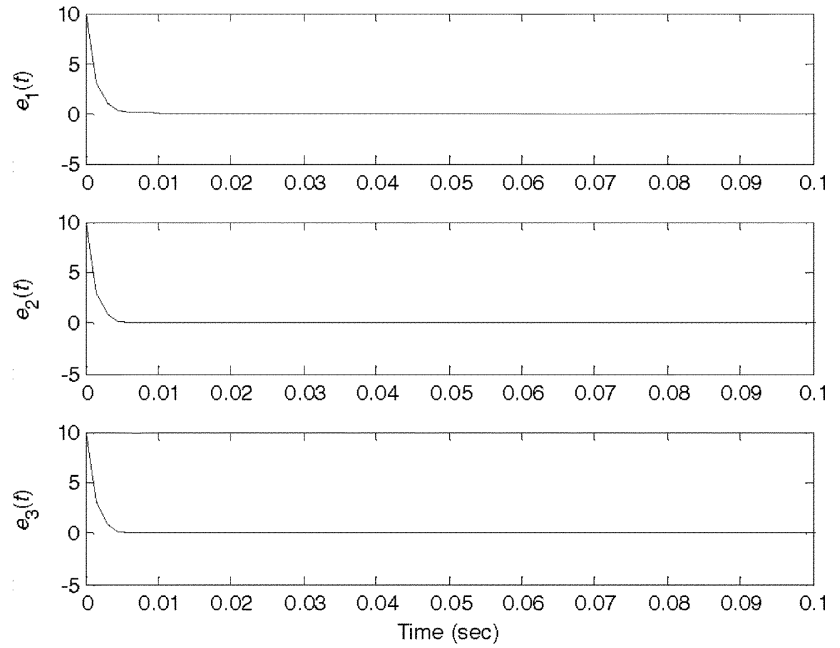

(b)

Fig. 11. Tracking error of the response Lorenz system with the proposed fuzzy controller. (a). Tracking error for $0 \leq \mathrm{t} \leq 15 \mathrm{~s}$. (b). Tracking error for $0 \leq$ $\mathrm{t} \leq 0.1 \mathrm{~s}$.

\section{CONCLUSION}

The synchronization of chaotic systems subject to parameter uncertainties using timed-delayed fuzzy state-feedback controller has been investigated. The fuzzy state-feedback controller using the system state of the response chaotic system and the time-delayed system state of the drive chaotic system has been proposed to realize the synchronization. To overcome the analysis difficulties introduced by the system time delay and parameter uncertainties, first, T-S fuzzy has been employed to represent the chaotic systems subject to parameter uncertainties. Then, the membership functions of both fuzzy model and fuzzy controller have been considered to facilitate the stability analysis and produce less conservative stability analysis result. LMI-based stability conditions have been derived using Lyapunov-based approach to guarantee the system stability and aid the design of the time-delayed fuzzy controller. Simulation examples have been given to illustrate the effectiveness of the proposed approach.

\section{REFERENCES}

[1] T. Takagi and M. Sugeno, "Fuzzy identification of systems and its applications to modeling and control," IEEE Trans. Syst., Man., Cybern. B, Cybern., vol. SMC-15, no. 1, pp. 116-132, Jan. 1985. 
[2] M. Sugeno and G. T. Kang, "Structure identification of fuzzy model," Fuzzy Sets Syst., vol. 28, pp. 15-33, 1988.

[3] K. Tanaka, T. Ikeda, and H. O. Wang, "A unified approach to controlling chaos via an LMI-based fuzzy control system design," IEEE Trans. Circuits Syst. I, Fundam. Theory Appl., vol. 45, no. 10, pp. 1021-1040, Oct. 1998.

[4] K. Y. Lian, C. S. Chiu, T. S. Chiang, and P. Liu, "LMI-based fuzzy chaotic synchronization and communications," IEEE Trans. Fuzzy Syst., vol. 9, no. 4, pp. 539-553, Aug. 2001.

[5] K. Y. Lain, P. Liu, T. C. Wu, and W. C. Lin, "Chaotic control using fuzzy model-based methods," Int. J. Bifurc. Chaos, vol. 12, no. 8, pp. 1827-1841, 2002.

[6] Y. W. Wang, Z. H. Guan, and H. O. Wang, "LMI-based fuzzy stability and synchronization of Chen's system," Phys. Lett. A, vol. 320, pp. 154-159, 2003.

[7] W. Jiang, Q. Dong, and D. Bin, "Observer-based robust adaptive variable universe fuzzy control for chaotic system," Chaos, Solitons, Fractals, vol. 23, pp. 1013-1032, 2002.

[8] C. W. Park, C. H. Lee, and M. Park, "Design of an adaptive fuzzy model based controller for chaotic dynamics in Lorenz systems with uncertainty," Inf. Sci., vol. 147, no. 1-4, pp. 245-266, 2002.

[9] J. H. Kim, C. W. Park, E. Kim, and M. Park, "Adaptive synchronization of T-S fuzzy chaoic systems with unknown parameters," Chaos, Solitons, Fractals, vol. 24, pp. 1353-1361, 2005.

[10] J. H. Kim, C. W. Park, E. Kim, and M. Park, "Fuzzy adaptive synchronization of uncertain chaotic systems," Phys. Lett. A, vol. 344, pp. 295-305, 2005.

[11] C. H. Hyun, J. H. Kim, E. Kim, and M. Park, "Adaptive fuzzy observer based synchronization design and secure communications of chaotic systems," Chaos, Solitons, Fractals, vol. 27, pp. 930-940, 2006.

[12] M. E. Yalçin, J. A. K. Suykens, and J. Vandewalle, "Master-slave synchronization of Lur'e systems with time-delay," Int. J. Bifurc. Chaos, vol. 11, no. 6, pp. 1707-1722, 2001.

[13] X. X. Laio and G. Chen, "Chaos synchronization of general Lur'e system via time-delay feedback control," Int. J. Bifurc. Chaos, vol. 13, no. 1, pp. 207-213, 2003.

[14] X. P. Guan, C. L. Chen, H. P. Peng, and Z. P. Fan, "Time-delayed feedback control of time-delay chaotic systems," Int. J. Bifurc. Chaos, vol. 13, no. 1, pp. 193-205, 2003.

[15] C. L. Chen, G. Feng, and X. P. Guan, "Robust synchronization of chaotic Lur'e system via delayed feedback control,' Phys. Lett. A, vol. 321, pp. 344-354, 2004

[16] Y. W. Wang, Z. H. Guan, and H. O. Wang, "LMI-based fuzzy stability and synchronization of Chen's system," Phys. Lett. A, vol. 320, pp. 154-159, 2003.

[17] J. Cao and J. Lu, "Adaptive synchronization of neural networks with or without time-varying delays," Chaos, vol. 16, no. 013133, pp. 1-6, 2006.

[18] J. Cao, P. Li, and W. Wang, "Global synchronization in arrays of delayed neural networks with constant and delayed coupling," Phys. Lett. A, vol. 353, pp. 318-325, 2006.

[19] W. Wang and J. Cao, "Synchronization in an array of linearly coupled networks with time-varying delay," Physica A, vol. 366, pp. 197-211, 2006.

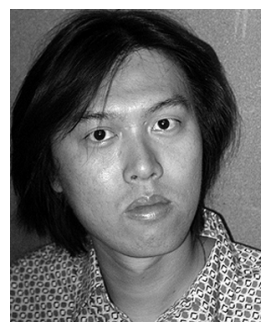

H. K. Lam (M'98) received the B.Eng. (Hons) and $\mathrm{Ph} . \mathrm{D}$. degrees from the Department of Electronic and Information Engineering, The Hong Kong Polytechnic University, Hong Kong, in 1995 and 2000, respectively.

From 2000 and 2005, he worked with the Department of Electronic and Information Engineering at The Hong Kong Polytechnic University as Post-Doctoral and Research Fellows, respectively. In 2005, he joined the King's College London, London, U.K., as a Lecturer. His current research interests include intelligent control systems and computational intelligence.

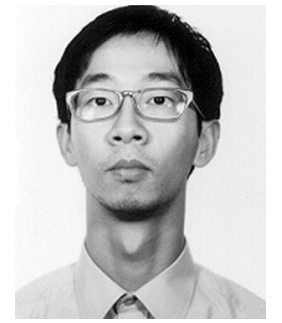

Wing-Kuen Ling received the B.Eng. (Hons) and M.Phil. degrees from the Department of Electrical and Electronic Engineering, the Hong Kong University of Science and Technology, in 1997 and 2000 , respectively, and the Ph.D. degree from the department of Electronic and Information Engineering from the Hong Kong Polytechnic University, in 2003.

In 2004, he joined the King's College London, London, U.K., as a Lecturer. His research interests include investigations of symbolic dynamics, applications of fuzzy and impulsive control theory, applications of functional inequality optimization theory, as well as filter banks and wavelets theory.

Dr. Ling has served as technical committee of several IEEE international fwrferences.

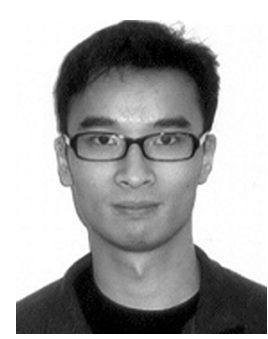

Herbert Ho-Ching Iu (S'98-M'00-SM'06) received the B.Eng. (Hons) degree in electrical and electronic engineering from the University of Hong Kong, Hong Kong, in 1997, and the Ph.D. degree from the Hong Kong Polytechnic University, Hong Kong, in 2000.

In 2002, he joined the School of Electrical, Electronic and Computer Engineering, The University of Western Australia, Perth, WA, Australia, as a Lecturer. In 2007, he became a Senior Lecturer. His research interests include power electronics, renewable energy, nonlinear dynamics and applications of chaos. He has published over 60 papers in these areas. He served as a Visiting Lecturer at the University of Reims Champagne-Ardenne, France, in 2004 and a Visiting Assistant Professor at the Hong Kong Polytechnic University, Hong Kong, in 2006. He currently serves as an Editorial Board Member for Australian Journal of Electrical and Electronics Engineering, and Guest Editor for Circuits, Systems and Signal Processing.

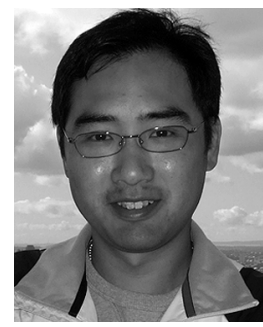

Steve S. H. Ling (''03-M'06) received the B.Eng. degree from the Department of Electrical Engineering, M.Phil. and Ph.D. degrees from the Department of Electronic and Information Engineering in the Hong Kong Polytechnic University in 1999, 2002 and 2006, respectively.

He is currently a Postdoctoral Research Associate in the School of Electrical, Electronic and Computer Engineering, University of Western Australia, Perth, WA, Australia. He has published over 45 research papers on Computational Intelligence and its industrial applications. His current research interests include evolution computations, fuzzy logics, neural networks, power systems, and recognitions. 